\title{
Correction to: Airplane pilot mental health and suicidal thoughts: a cross-sectional descriptive study via anonymous web- based survey
}

Alexander C. Wu' ${ }^{1}$, Deborah Donnelly-McLay ${ }^{1}$, Marc G. Weisskopf ${ }^{1}$, Eileen McNeely ${ }^{1}$, Theresa S. Betancourt ${ }^{2}$ and Joseph G. Allen ${ }^{1,3^{*}}$

\section{Correction}

After publication of the article [1], it has been brought to our attention that the specificity of the tool was reported as $88 \%$. This specificity is for Major Depressive Disorder. The specificity of the tool for all depressive disorders, the outcome of interest in the study, is $93 \%$.

\footnotetext{
Author details

'Department of Environmental Health, Harvard T.H. Chan School of Public Health, 665 Huntington Avenue, Building 1, Room 1301, Boston, MA 02115, USA. ${ }^{2}$ Department of Global Health and Population, Harvard T.H. Chan School of Public Health, 665 Huntington Avenue, Building 1, Room 1104 Boston, MA 02115, USA. ${ }^{3}$ Harvard T.H. Chan School of Public Health, 401 Park Drive, Landmark Center, 404-L, Boston, MA 02215, USA.
}

Received: 10 November 2017 Accepted: 14 November 2017 Published online: 27 November 2017

\section{Reference}

1. Wu A, Donnelly-McLay D, Weisskopf M, McNeely E, Betancourt T, Allen J. Airplane pilot mental health and suicidal thoughts: a cross-sectional descriptive study via anonymous web-based survey. Environ Health. 2016; 15(1):121. https://doi.org/10.1186/s12940-016-0200-6.

\footnotetext{
* Correspondence: JGAllen@hsph.harvard.edu

${ }^{1}$ Department of Environmental Health, Harvard T.H. Chan School of Public Health, 665 Huntington Avenue, Building 1, Room 1301, Boston, MA 02115, USA

${ }^{3}$ Harvard T.H. Chan School of Public Health, 401 Park Drive, Landmark Center, 404-L, Boston, MA 02215, USA

Full list of author information is available at the end of the article
} 